DOI:

\title{
THE ENGAGEMENT OF ANALOG AND DIGITAL APPROACHES IN TEACHING TRANSLATION
}

\author{
Nataliya Gavrilenko \\ Doctor of Pedagogical sciences, Professor \\ Peoples' Friendship University of Russia - RUDN University \\ (Moscow, Russia) \\ e-mail: nngavrilenko@narod.ru
}

\begin{abstract}
The article discusses the current state of digital competence of a translator. For this purpose, the analysis of the competences allocated by researchers associated with the use of information technology in the activities of the translator has been carried out. The results of the foresight session "Translator 2030" have been analysed, and the status of the draft project of the professional standard of the translator has been reviewed. This interdisciplinary integrated document comprehensively describes this complex professional activity, which today is associated with the active use of digital technology. In the draft of the professional standard, four generalized labor functions of a translator are highlighted: technological support for translation activities, non-specialized translation, professionally oriented translation, translation management. The analysis made it possible to present the composition of the digital competence of the translator, which includes technical, informational, organizational and communication components.
\end{abstract}

Keywords: digital competence, key competence of a translator, draft professional standard, information technology

\section{ОБРУЧЕНИЕ АНАЛОВОГО И ЦИФРОВОГО ПОДХОДОВ ПРИ ОБУЧЕНИИ ПЕРЕВОДУ}

\author{
Наталия Гавриленко \\ д.п.н., проф. кафедры иностранных языков \\ Инженерная академия \\ Российский университет дружбы народов \\ (Москва, Россия) \\ e-mail: nngavrilenko@narod.ru
}

\begin{abstract}
Аннотация: В статье рассматривается современное состояние цифровой компетентности переводчика. С этой целью проведён анализ выделяемых исследователями компетенций, связанных с использованием информационных технологий в деятельности переводчика. Проанализированы результаты форсайт-сессии «Переводчик 2030», и рассмотрен состояние разрабатываемыйпроект профессионального стандарта переводчика. Данный междисциплинарный интегрированный документ всесторонне описывает эту сложную профессиональную деятельность, которая сегодня связана с активным использованием цифровых технологий. В проекте профстандарта выделены четыре обобщенные трудовые функции переводчика: технологическая поддержка переводческой деятельности, неспециализированный перевод, профессионально ориентированный перевод, руководство переводческой деятельности. Проведенный анализ позволил представить состав цифровой компетентности переводчика, которая включает технический, информационно-организационный и коммуникационный компоненты.
\end{abstract}

Ключевые слова: цифровая компетентность, ключевые компетентности переводчика, проект профессионального стандарта, информационные технологии

ВВЕДЕНИЕ. Сегодня практически каждый специалист должен уметь создавать и обрабатывать сложную информацию думать системно и критически, принимать решения на многокритериальной основе понимать суть происходящих процессов полидисциплинарного характера быть адаптивным и гибким к новой информации быть креативными, уметь выявлять и решать реальные проблемы цифрового мира. «Возникают не только новые цифровые профессии и исчезают старые аналоговые, но и внезапно появляются потребности в кадрах в таких объемах, которых просто нет на рынках трудах» (Kupriyanovskiy et. al., 2017: 21). Информационные технологии являются важнейшим ядром цифровой среды, и в процессе своей деятельности переводчик должен не просто изолированно изучать цифровую среду, а обязательно сотрудничать со специалистами, разрабатывающими эти технологии, что подразумевает «гибридную», междисциплинарную суть профессии переводчика. «Цифровая среда - это понятие, которое основано на наличии некоторого количества концептов, имеющих не гуманитарное измерение, а естественно-научное или математическое. Если мы спросим, что такое цифровая среда как явление, то мы можем ее довольно просто описать через три понятия - это данные, которые закодированы в двоичной системе, это алгоритмы, это программное обеспечение» (Moroz 2017).

В нашу жизнь входят такие понятия, как циирровая среда, цифровая грамотность, ичфровое сообщество, цифрровая экономика, цифрровизация образования. Цифровизация деятельности переводчика ведет к изменениям на рынке труда, в профессиональном стандарте, к выявлению новых компетентностей переводчика, а следовательно, и к переосмыслению образовательных стандартов и путей подготовки профессиональных переводчиков.

Цифровые ресурсы, которые переводчик может использовать сегодня в своей профессиональной деятельности, позволяют преодолевать традиционные взгляды на перевод и на скорость выполнения этой сложной деятельности. «Цифровые технологии, с одной стороны, способствуют дальнейшему повышению объемов и 
эффективности производства, с другой - позволяют реализовывать индивидуальный подход в различных сферах» (Nikulina and Starichenko, 2018: 108).

ВЛИЯНИЕ ЦИФРОВЫХ ТЕХНОЛОГИЙ НА ДЕЯТЕЛЬНОСТЬ ПЕРЕВОДЧИКА. Переводческое сообщество рассматривает различные сценарии развития профессии переводчика. Предполагается увеличение потребности в переводе с таких языков, как японский, китайский и арабский, появление междисциплинарных профессий, отмечается увеличение потребности в переводе аудиовизуального контента (About, 2017).C развитием технологий предполагается рост спроса на перевод, осуществляемый переводчиками-фрилансерами, а не представителями переводческих компаний (Quelle société de traduction pour le futur? 2017).

Отмечаются и такие негативные тенденции, как «Уберизация» профессии переводчика. Использование платформам Stepes, Netflix, Hermes позволяет переводчику переводить выложенные предприятиями тексты прямо со своего смартфона, что обусловливает низкие тарифы на перевод. Отмечается также, что использование информационных технологий ведет к снижению ответственности переводчика за конечный результат (Моrеau, 2017).

Вместе с тем цифровизация оказывает и положительное влияние на развитие переводческой отрасли. Например, канадские исследователи видят в современном информационном обществе увеличение роли переводав сохранении культурной и национальной идентичности (Korchagina, 2016). В российском переводческом сообществе в мае 2017 г. «Школой дидактики перевода» была проведена «Форсайт-сессия: Переводчик 2030», позволившая наметить возможные пути развития этой сложной профессии (www.gavrilenko-nn.ru). Главным трендом проведенной сессии был единодушно назван тренд «Автоматизаџия функции перевода вследствие развития высоких технологий». В рамках данного тренда участники выделили следующие мини-тренды:

- активное внедрение роботов-переводчиков и систем полуавтоматического перевода (Trados, Smartcat, CATLabs и др.);

- внедрение и развитие технологии machinelearning, начало обработки больших массивов данных (BigData) и их применение в переводческой сфере;

- постоянное совершенствование автоматизированных переводческих алгоритмов;

- «геймификация» переводческого функционала;

- резкое увеличение нетекстового контента, для передачи которого переводчики должны обладать новыми умениями и навыками;

- рост доли машинного перевода.

В рамках рассмотрения тренда «Автоматизачия функции перевода вследствие развития высоких технологий» активно обсуждались угрозы, к которым участники форсайта отнесли:

- снижение потребности в «человеческом» переводе;

- падение доходов классических переводчиков;

- профессиональную деградацию: чем больше смогут делать роботы, тем меньше будут уметь сами переводчики;

- слабую подготовленность переводчиков к лавиноподобному развитию программного обеспечения;

- уровень профессиональной компетентности переводчика начинают анализировать машины-роботы, что приводит к риску ошибочной оценки;

- отставание уровня подготовки переводчиков в области использования программного обеспечения от потребностей рынка.

Вместе в тем были отмечены и возможности, которые предоставляет данный тренд:

появление новых смежных/междисциплинарных профессий или дополнительного функционала у переводчиков;

возвращение востребованности креативности и когнитивных навыков и умений; 2018).

необходимость в высокопрофессиональных переводчиках для обучения роботов. (Gavrilenko and Biryukova,

Проведенная форсайт-сессия «Переводчик 2030» послужила началом разработки профессионального стандарта переводчика, которая ведется в России уже более двух лет. Представлен междисциплинарный интегрированный документ, всесторонне описывающий эту сложную профессиональную деятельность. Анализ деятельности предприятий, оказывающих переводческие услуги, показал, что работодателям приходится доучивать переводчиков, организовывая специальные курсы. Во многом это связано с активным использованием цифровых технологий в деятельности переводчика, что сильно повлияло на разрабатываемый проект профстандарта. В результате в последнем варианте данного проекта были выделены четыре обобщенные трудовые функциипереводчика:

- технологическая поддержка переводческой деятельности: технологическое планирование переводческого проекта; подготовка исходных текстов для перевода и оформление текста перевода; технологическое обеспечение переводческих и локализационных проектов с применением систем управления содержимым; подготовка исходных текстов для машинного и автоматизированного перевода, предперевод; терминологическое сопровождение перевода; обработка памяти переводов;

- неспечиализированный перевод: устный сопроводительный перевод; письменный перевод типовой документации;

- профессионально ориентированный перевод: устный последовательный перевод; синхронный перевод; письменный перевод (в том числе с использованием специализированных инструментальных средств); 
художественный перевод; локализация аудиовизуальных произведений; аудиовизуальный перевод для аудиторий с особыми когнитивными потребностями;

- руководство переводческой деятельности: редакторский контроль перевода; предоставление консультаций по улучшению качества перевода; управление производственным процессом перевода.

Описание данных обобщенных трудовых функций в последнем проекте профессионального стандарта «Специалист в области перевода» обусловило появление следующих новых специальностей, связанных с использованием цифровых технологий: верстальщик перевода, переводчик-технолог, постредактор машинного перевода.

Разработка проекта профессионального стандарта «Специалист в области перевода» открывает новые возможности перед преподавателями вузов при создании образовательных стандартов. Действия, знания и умения, необходимые для осуществления трудовых функций переводчика, представленные в профстандарте, возможно включать в процесс формирования профессиональной компетентности переводчика.

ЦИФРОВАЯ КОМПЕТЕНТНОСТЬ ПЕРЕВОДЧИКА И ЕЁ КОМПОНЕНТЫ. ПроведеннЫЙ анализ состояния профессиональной деятельности переводчика показывает, что раньше переводчики имели дело только с аналоговой информацией, которую воспринимали с помощью органов чувств: слуха, зрения, обоняния и т.д. Теперь в их профессию вошли цифровые устройства: компьютеры, мобильные телефоны, проигрыватели компакт-дисков и т.д. Данный вопрос представляется важным при подготовке переводчиков, поскольку переводчик, как и любой человек, привык работать с аналоговой информацией, тогда как вычислительная техника работает с цифровой информацией.

Основное различие между аналоговым и цифровым сигналом заключается в структуре передаваемого сигнала. Аналоговые сигналы представляют непрерывный поток колебаний с изменяющимися амплитудой и частотой. Их преимуществом является то, что именно в аналоговом виде мы воспринимаем звук своими ушами. Недостаток заключается в невозможности хранения, передачи и тиражирования сигнала.

Цифровой сигнал представляет собой дискретные колебания, значения которых зависят от передающей среды, и воспринимается вычислительной техникой. Важным преимуществам цифрового сигнала является точность при копировании и передаче звукового потока. К недостатком цифрового сигнала принято считать то, что данный сигнал выступает промежуточной стадией, и для восприятия этого сигнала человеком требуется цифро-аналоговый преобразователь, который переводит цифровой звук в аналоговый.

Необходимость работать не только с аналоговой, но и цифровой информацией, находить в сети необходимую информацию, работать с терминологическими базами данных, общаться в сети с заказчиком, создавать текст перевода в соответствующем формате и т.д. позволила исследователям выделять соответствующую компетенцию переводчика. Рассматривая данную компетенцию переводчика, исследователи называют её инструментальной (РАCTE, 2007), технической (Porshneva, 2004), информационно-технологической (Alferova, 2010), информационноорганизационной (Leonova, 2017) и т.д.

В обновленном варианте требований к переводческой компетентности на сайте Европейского Союза представлена технологическая компетенция, которая включает следующий перечень знаний и умений переводчика:

- продуктивно использовать необходимые компьютерные приложения, включая полный спектр офисного программного обеспечения, и быстро адаптироваться к новым инструментам и новым цифровым ресурсам;

- эффективно использовать поисковые системы, инструменты на основе корпусов, инструменты текстового анализа и инструменты перевода;

- готовить, обрабатывать и управлять файлами и другими медиаисточниками как частью процесса перевода, например видео и мультимедиа,;

- осваивать основы машинного перевода и его влияние на процесс перевода;

- оценивать релевантность систем машинного перевода при переводе и при необходимости внедрять соответствующую систему в процесс перевода;

- применять другие инструменты для осуществления перевода, такие как программное обеспечение для управления рабочими процессами (Référentiel.., 2017).

Несмотря на различные названия компонентный состав данной компетенции, выделяемый исследователями, остается примерно одинаковым. Однако в выделяемых компетенциях не учтены все стороны современной деятельности переводчика в цифровом мире. В 2017 г. Комиссией по широкополосной связи в интересах устойчивого развития (Broadband Commission for Sustainable Development, основана ЮНЕСКО и Международным союзом электросвязи) был представлен отчет «Цифровые навыки для жизни и работы», который определил три группы цифровых навыков и компетенций современного человека:

- «базовые функциональные навыки», которые необходимы для получения доступа к началу работы с цифровыми технологиями;

- «стандартные цифровые навыки», которые необходимыдля осознанного использования цифровых технологий;

- «продвинутые навыки», которые необходимы для расширения и «преобразования» использования циифровых технологий (Doklad sovmestnoy..., 2017).

Как показали проведенная форсайт-сессия и анализ исследований переводоведов, выделенные навыки в полной мере представлены и в профессии переводчика, который не просто изолированно изучает необходимые для работы информационные технологии, но и обязательно сотрудничает со специалистами, которые разрабатывают эти технологии. Данный подход объясняет «гибридную», междисциплинарную суть современной профессии переводчика. Конечно, важно, чтобы переводчик знал, как устроена цифровая среда на технологическом уровне, но, кроме этого, он должен понимать меняющийся опыт человечества в области цифры и учитывать возможности, 
которые данная среда открывает для его профессионального роста. Переводчику также важно помнить, что цифровое пространство является публичным, что подразумевает аккуратное отношение переводчика к использованию и распространению любых данных, любой информации. Ответственность переводчика включает умение обеспечивать онлайн-безопасность при работе с информацией в интернете. Иными словами цифровая среда для переводчика - это среда, в которой он должен быть успешен, результативен, и от того, как он представлен в этой среде, во многом зависит его профессиональная карьера.

Проведенный анализ позволил выделить в качестве одной из ключевых компетентностей (подробный анализ ключевых компетентностей см.: Gavrilenko, 2018) - цифровую компетентность переводчика, которая представляет собой готовность, способность и ответственность эффективно, критично и безопасно выбирать и применять информационные технологии на всех этапах своей профессиональной деятельности. Выделяемые исследователями знания и умения переводчика в области информационных технологий были сгруппированы в рамках следующих составляющих цифровой компетентности переводчика:

- Техническая составляющая циифровой компетентности переводчика представляет способность/готовность переводчика использовать умения безопасно работать в сети и эффективно применять программное обеспечение при решении стоящих пред ним профессиональных задач на всех этапах своей деятельности и будет включать определенные знания и умения.

- Информационно-организационная составляющая цифровой компетентности переводчикас вязана со способностью/готовностью переводчика использовать систему знаний умений, личностных качеств, необходимую для поиска, обработки, представления, хранения полученной информации и для организации в сети своей профессиональной деятельности (Леонова, 2016).

- Коммуникационная составляющая цифровой компетентности переводчика представляет способность/готовность и ответственность переводчика мобилизовать свои умения и знания для безопасного осуществления онлайн-коммуникации в различных формах (электронная почта, чаты, блоги, форумы, социальные сети и др.) (Gavrilenko, 2018).

Остается открытым вопрос, в какой последовательности целесообразно формировать компоненты цифровой компетентности переводчика. Среди основных направлений применения Интернета в переводческой практике исследователи выделяют 4 блока: поиск и получение заказов, коммуникация с коллегами, работа с переводческими онлайн-программами, поиск информации для решения переводческих задач (Korol'kova et. al., 2018). Деятельностный подход позволил Д.А. Алфёровой разработать интегративную модель формирования информационной компетентности переводчика текстов по специальности, в рамках которой автор предложила алгоритм использования информационных технологий, начиная с первых шагов переводчика - поиска работы, беседы с заказчиком и получения заказа до момента сдачи отредактированного перевода его заказчику (Alferova, 2010).Такой деятельностный подход к формированию цифровой компетентности переводчика позволяет научить студентов использовать возможностями цифровой среды на всех этапах своей работы.

ЗАКЛЮЧЕНИЕ. Проведенный анализ показывает значимость цифровой компетентности переводчика, необходимость её выделения в качестве одной из ключевых составляющих профессионализма переводчика. Выделение в рамках данной компетентности технического, информационно-организационного и коммуникационного компонентов показывает необходимость знаний не только технической стороны использования цифровых технологий при переводе, но и умений соблюдать безопасность при использовании цифровой информации и этической стороны при общении в сети. В процессе подготовки переводчиков формирование данной компетентности влечёт за собой необходимость разработки соответствующей методики.

Разработка данной методики представляется достаточно сложным и трудоёмким процессом. Выделяемые знания и умения по своей сути находятся на стыке гуманитарных и технических наук, их возможно формировать только с междисциплинарных позиций. Количество важной для переводчика цифровой информации неизменно растет и будут предлагаться всё новые возможности её обработки. Поэтому знания и умения цифровой компетентности переводчика должны регулярно корректироваться, адаптироваться к новым условиям с учётом динамики процессов цифровой экономики, что значительно усложняет деятельность преподавателя, так как требует постоянного обновления программ. Данный вопрос должен решаться с привлечением практикующих переводчиков и разработчиков программного переводческого обеспечения. Формирование цифровой компетентности переводчика целесообразно осуществлять не изолированно, а поэтапно в процессе решения стоящих перед переводчиком задач.

\section{LIST OF REFERENCES}

Alferova D.A. (2010). Modul'noye obucheniye perevodu nauchno-tekhnicheskikh tekstov s ispol'zovaniyem informatsionnykh tekhnologiy [Modular training in the translation of scientific and technical texts using information technologies]: дисс. ... кандидата педагогических наук. Москва, 273.

Gavrilenko N.N. (2018). Klyuchevyye kompetentnosti perevodchika [Key Competencies of a Translator] // Didaktika perevoda: traditsii i innovatsii: kollektivnaya monografiya / Pod red. N.N. Gavrilenko. M.: Flinta, 27-59.

Gavrilenko N.N., Biryukova Y.N. (2018). Razvitiye professi i perevodchika: instrument prognozirovaniya (na primere forsayt-tekhnologii) [Development of the translator's profession: forecasting tools (by the example of foresight technology)] // Vestnik «Zdorov'ye i obrazovaniye v KHKHI veke. 20, № 7, 18-24.

Doklad sovmestnoy komissii YUNESKO i MSE o tsifrovykh navykakh, neobkhodimykh «dlya zhizni i raboty» [Report of the joint commission of UNESCO and ITU on the digital skills necessary for "living and working"], oktyabr', 2017, http://d-russia.ru [6.11.2017].

Korol'kova S.A., Novozhilova A.A., Sheyko A.M., ShovgeninaYe.A. (2018). Intrenet-resursyi SAT-sistemy v perevode [Internet resources and CAT systems in translation: Ucheb.-metod. Posobiye s CD-prilozheniyem. Angliyskiy, nemetskiy, frantsuzskiyyazyki, M.: «R.Valent»,124. 
Kupriyanovskiy V.P., Sukhomlin V.A., Dobrynin A.P., Raykov A.N., Shkurov F.V., Drozhzhinov V.I., Fedorova N.O., Namiot D.Ye. (2017). Navyki v tsifrovoy ekonomikei vyzov y sistemy obrazovaniya [Digital Skills and the Challenges of the Education System] // International Journal of Open Information Technologies. vol. 5, 1, 19-25.

Leonova A.S. (2016). Razvitiye informatsionno-organizatsionnoy kompetentsii budushchego perevodchika [Development of the informational and organizational competence of the future translator]: Diss. ... kand.ped.nauk. - Kazan', 218.

Moroz O. (2017). Normy tsifrovoy gramotnosti [Norms of digital literacy] // PostNauka. 9 yanvarya 2017. https://postnauka.ru/video/ 7200. [ 5.11.2017].

Nikulina V.N., Starichenko Ye.B. (2018). Informatizatsiya i tsifrovizatsiya obrazovaniya: ponyatiya, tekhnologii, upravleniye [Informatization and digitalization of education: concepts, technologies, management] // Pedagogicheskoye obrazovaniye v Rossii. № 8 , 107114.

Porshneva Ye.R. (2004) Mezhdistsiplinarnyye osnovy bazovoy lingvisticheskoy podgotovki spetsialista-perevodchika: diss.dokt.pednauk. Kazan', 425.

About K. (2017). Traducteur, un métier d'avenir http://redaction-articles.com/traducteur-un-metier-davenir [10.12.17].

Korchagina E. (2017). Le futur de la traduction au Canada, 2016 http://www.artsrn.ualberta.ca/lesfrancopains/?page id=523[10.12.17].

Moreau A. (2017). Le métier de Traducteur à l'heure de l'uberisation de l'économie

https://mastertsmlille.wordpress.com/2017/05/08/le-metier-de-traducteur-a-lheure-de-luberisation-de-leconomie/ [10.12.17].

PACTE. (2007). Une recherche empirique expérimentale sur la competence de traduction. Traduction spécialisée. Quelle qualification pour les traducteurs? / Direction : Daniel Gouadec. Rennes : La Maison du dictionnaire, 95-116

Quelle société de traduction pour le futur? (2017) https://lexling.com/blog/quelle-agence-de-traduction-futu[10.12.17].

Référentiel de compétences de L'EM-IExpert Group - 2017

https://ec.europa.eu/info/sites/info/files/emt_competence_fwk_2017_en_web.pdf [16.02.2018].

\title{
For citation:
}

Gavrilenko Nataliya (2019) THE ENGAGEMENT OF ANALOG AND DIGITAL APPROACHES IN TEACHING TRANSLATION // International Scientific-Pedagogical Organization of Philologists “ WEST-EAST" (ISPOP). Scientific Journal WEST-EAST. Vol 2/1 N1 (October, 2019). p. 130-134. doi:

\section{Для цитирования:}

Гавриленко Наталия (2019) ОБРУЧЕНИЕ АНАЛОВОГО И ЦИФРОВОГО ПОДХОДОВ ПРИ ОБУЧЕНИИ ПЕРЕВОДУ // Internationa 1Scientific-Pedagogical Organization of Philologists “ WEST-EAST” (ISPOP) . Scientific Journal WEST-EAST. Vol 1/1 N1 (October, 2019). C. 130-134. doi:

\section{Information about the author:}

Nataliya Gavrilenko - Doctor of Pedagogical sciences, Professor Foreign Languages Department Engineering Academia, Peoples' Friendship University of Russia - RUDN University,Moscow, Russia.

e-mail: nngavrilenko@narod.ru

Сведения об авторе: Наталия Гавриленко - д.п.н., проф. кафедры иностранных языков

Инженерная академия, Российский университет дружбы народов, Москва, Россия.

e-mail: nngavrilenko@narod.ru

Manuscript received: 05/20/2019 Accepted for publication: 09/20/2019

DOI:

\section{AUDIOFILES WITH ORAL FAMILY COMMUNICATION IN THE PRACTICE OF TEACHING RUSSIAN AS A FOREIGN LANGUAGE}

\author{
Yuliya Pikuleva \\ PhD in Philology, associate professor, \\ Department of Russian language, General linguistics and speech communication at \\ Ural Federal University named after the first President of Russia B.N. Yeltsin \\ (Yekaterinburg, Russia) \\ e-mail: pik@bk.ru
}

\begin{abstract}
The article is devoted to the description of the methodology used in the process of speech training of foreign undergraduates and graduate students in UrFU. To increase the level of proficiency students at advanced levels of training are offered not just listening colloquial speech, but a more complicated procedure - transcript of audio taken with colloquial conventional signs. Regular work on listening and decoding oral family communication allows foreign students to understand its thematic, stylistic and value specifics, to easier interpret the purpose of the spoken text, to introduce new words and expressions into speech practice of each student. Commenting on the transcripts by the teacher contains an interpretation of deviations from the norms. Typical problem areas in listening are identified: significant for family communication precedent expressions, unofficial family names, language-game. The use of the proposed method can improve the linguistic, communicative and cultural competence of foreign students.
\end{abstract}

Key words: Russian as a foreign language, listening, unadapted text, family communication 